\title{
Shot-gather time migration of planar reflectors without velocity model
}

\author{
Andrej Bóna ${ }^{1}$
}

\begin{abstract}
Standard migration techniques require a velocity model. A new and fast prestack time migration method is presented that does not require a velocity model as an input. The only input is a shot gather, unlike other velocityindependent migrations that also require input of data in other gathers. The output of the presented migration is a time-migrated image and the migration velocity model. The method uses the first and second derivatives of the traveltimes with respect to the location of the receiver. These attributes are estimated by computing the gradient of the amplitude in a shot gather. The assumptions of the approach are a laterally slowly changing velocity and reflectors with small curvatures; the dip of the reflector can be arbitrary. The migration velocity corresponds to the root mean square (rms) velocity for laterally homogeneous media for near offsets. The migration expressions for $2 \mathrm{D}$ and $3 \mathrm{D}$ cases are derived from a simple geometrical construction considering the image of the source. The strengths and weaknesses of the methods are demonstrated on synthetic data. At last, the applicability of the method is discussed by interpreting the migration velocity in terms of the Taylor expansion of the traveltime around the zero offset.
\end{abstract}

\section{INTRODUCTION}

It is possible to find the velocity and the reflector below a constant velocity layer by using traveltimes and horizontal slownesses, also called local event slopes, of the reflected rays. According to Sword (1987), the idea of using horizontal slownesses to obtain migration velocity and the location goes back to Puzyrev, Riznichenko, and Rudnev in the 1940s. This approach was published in English by Hermont (1979), Sword (1981), Zavalishin (1982), and Goldin (1986). Sword (1987) presents this constant-velocity prestack depth migration alongside his tomography approach. The prestack time migration proposed by Fomel (2007) is equivalent to these migrations because he assumes a constant effective-velocity layer, which means that the traveltime curves are hyperbolas. The idea of using horizontal slowness was also used by Kleyn (1977) for zero-offset migration and by Ottolini (1983) for migration of horizontal reflectors. Cooke et al. (2009) present another point of view on this velocity-independent migration in which they use the resulting migration velocity to perform multiples suppression. All of these formulations require the horizontal slownesses in two domains: some in common-offset and common-midpoint (e.g., Fomel, 2007) and some in common-shot and common-receiver domains (e.g., Cooke et al., 2009). However, the information about the slowness is not always available in two domains; for example, the spacing between sources can be too large or there are only few sources available.

To address this issue, I formulate a new method for velocityindependent migration that requires data only in one domain; it uses the first and the second derivatives of the traveltime with respect to the location of the receiver in a common-shot gather. As with the other methods, I assume that the signal propagates through a constant effective-velocity layer; that is, the traveltime curves are hyperbolic, and the velocity is given by the secondorder term in the Taylor expansion of the traveltime around the zero offset. This assumption is a good approximation to many geological situations, including the vertical inhomogeneity, as indicated by the success of time migrations. Moreover, I assume that the reflector has a small curvature (compared with its depth) with an arbitrary dip. First, I describe the theory of the migration algorithm. Second, I illustrate the applications and limitations of the method on three synthetic examples. The appendix shows the generalization of the method to $3 \mathrm{D}$.

\section{THEORY}

In this section I describe the shot-gather migration in a $2 \mathrm{D}$ medium. If $t$ denotes the traveltime of a signal from a fixed source with location given by coordinates $\left(x_{s}, z_{s}\right)$ to a receiver

Manuscript received by the Editor 13 December 2009; revised manuscript received 25 October 2010; published online 10 March 2011.

${ }^{1}$ Curtin University, Department of Exploration Geophysics, Kensington, Western Australia, Australia. E-mail: a.bona@curtin.edu.au. (C) 2011 Society of Exploration Geophysicists. All rights reserved. 
located at $\left(x_{r}, z_{r}\right)$, then the horizontal slowness at the receiver is defined as

$$
p_{x}=\frac{\partial t}{\partial x_{r}}
$$

If the wave propagates through a constant effective-velocity medium with slowness $p=\frac{1}{v}$, where $v$ is the effective velocity, then one can find the reflection point by finding the reflected image $\left(x_{s^{\prime}}, z_{s^{\prime}}\right)$ of the source around the reflection plane (Figure 1). The location of this image is such that its distance from the receiver is equal to $\frac{t}{p}$ and the angle $\alpha$ between the line $z=z_{r}$ and the line connecting the receiver with the image is $\arccos \left(\frac{p_{x}}{p}\right)$; its coordinates are given by

$$
z_{s^{\prime}}=z_{r}+\frac{t}{p} \sqrt{1-\left(\frac{p_{x}}{p}\right)^{2}}=z_{r}+\frac{t}{p^{2}} \sqrt{p^{2}-p_{x}^{2}}
$$

and

$$
x_{s^{\prime}}=x_{r}-\frac{t p_{x}}{p^{2}} .
$$

As illustrated by Figure 1, the reflection point $(x, z)$ is located at the intersection of the line passing though the receiver and the image of the source, indicated by the green dashed line,

$$
\left(z_{s^{\prime}}-z_{r}\right)\left(x-x_{r}\right)=\left(x_{s^{\prime}}-x_{r}\right)\left(z-z_{r}\right),
$$

with the line that is normal to the source-reflected source line and passes through the middle of this line, indicated by the black dotted line,

$$
\left(x-\frac{x_{s^{\prime}}+x_{s}}{2}\right)\left(x_{s^{\prime}}-x_{s}\right)+\left(z-\frac{z_{s^{\prime}}+z_{s}}{2}\right)\left(z_{s^{\prime}}-z_{s}\right)=0 .
$$

The coordinates of the reflection point are the solution of equations 4 and 5 and are given by

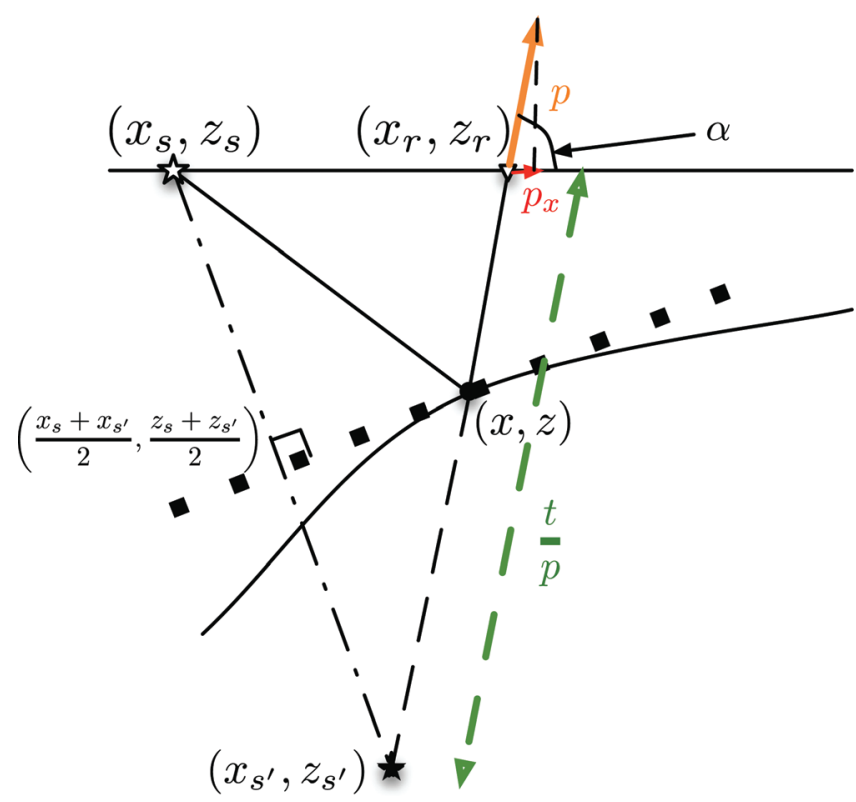

Figure 1. Reflected source (star) as "viewed" from the receiver (triangle).

$$
\begin{aligned}
x= & \frac{x_{r}\left(x_{s}^{2}-x_{s^{\prime}}^{2}+\left(z_{s}-z_{s^{\prime}}\right)^{2}\right)}{2\left(\left(x_{r}-x_{s^{\prime}}\right)\left(x_{s}-x_{s^{\prime}}\right)+\left(z_{r}-z_{s^{\prime}}\right)\left(z_{s}-z_{s^{\prime}}\right)\right)} \\
& +\frac{x_{s^{\prime}}\left(x_{s^{\prime}}^{2}-x_{s}^{2}+z_{s^{\prime}}^{2}-z_{s}^{2}+2 z_{r}\left(z_{s}-z_{s^{\prime}}\right)\right)}{2\left(\left(x_{r}-x_{s^{\prime}}\right)\left(x_{s}-x_{s^{\prime}}\right)+\left(z_{r}-z_{s^{\prime}}\right)\left(z_{s}-z_{s^{\prime}}\right)\right)}
\end{aligned}
$$

and

$$
\begin{aligned}
z= & \frac{2\left(x_{r} x_{s^{\prime}} z_{s^{\prime}}-x_{s} x_{s^{\prime}} z_{r}-x_{r} x_{s^{\prime}} z_{s^{\prime}}\right)}{2\left(\left(x_{r}-x_{s^{\prime}}\right)\left(x_{s}-x_{s^{\prime}}\right)+\left(z_{r}-z_{s^{\prime}}\right)\left(z_{s}-z_{s^{\prime}}\right)\right)} \\
& +\frac{x_{s^{\prime}}^{2}\left(z_{r}+z_{s^{\prime}}\right)+\left(z_{r}-z_{s^{\prime}}\right)\left(x_{s}^{2}+z_{s}^{2}-z_{s^{\prime}}^{2}\right)}{2\left(\left(x_{r}-x_{s^{\prime}}\right)\left(x_{s}-x_{s^{\prime}}\right)+\left(z_{r}-z_{s^{\prime}}\right)\left(z_{s}-z_{s^{\prime}}\right)\right)} .
\end{aligned}
$$

Substituting for $x_{s^{\prime}}$ and $z_{s^{\prime}}$ from expressions 2 and 3 into expressions 6 and 7, and expressing $z$ in terms of the two-way vertical traveltime $t_{0}=2 z p$ gives

$$
\begin{aligned}
x= & \frac{2 p^{2} x_{r}\left(t+\sqrt{p^{2}-p_{x}^{2}}\left(z_{r}-z_{s}\right)\right)}{2 p^{2}\left(t+p_{x}\left(x_{s}-x_{r}\right)+\sqrt{p^{2}-p_{x}^{2}}\left(z_{r}-z_{s}\right)\right)} \\
& +\frac{p_{x}\left(p^{2}\left(x_{s}^{2}-x_{r}^{2}+\left(z_{r}-z_{s}\right)^{2}\right)-t^{2}\right)}{2 p^{2}\left(t+p_{x}\left(x_{s}-x_{r}\right)+\sqrt{p^{2}-p_{x}^{2}}\left(z_{r}-z_{s}\right)\right)}
\end{aligned}
$$

and

$$
\begin{aligned}
t_{0}= & \frac{\sqrt{p^{2}-p_{x}^{2}}\left(t^{2}-p^{2}\left(\left(x_{r}-x_{s}\right)^{2}+z_{s}^{2}-z_{r}^{2}\right)\right)}{p\left(t+p_{x}\left(x_{s}-x_{r}\right)+\sqrt{p^{2}-p_{x}^{2}}\left(z_{r}-z_{s}\right)\right)} \\
& +\frac{2 p^{2} z_{r}\left(t-p_{x}\left(x_{r}-x_{s}\right)\right)}{p\left(t+p_{x}\left(x_{s}-x_{r}\right)+\sqrt{p^{2}-p_{x}^{2}}\left(z_{r}-z_{s}\right)\right)} .
\end{aligned}
$$

To use these expressions, it is necessary to know the effective slowness $p$ of the medium. One can find this slowness if one assumes that the reflector can be approximated by a plane. In such a case, the location of the image of the source does not change if the location of the receiver is infinitesimally changed, as illustrated in Figure 2. In other words, the derivative of equations 2 and 3 with respect to $x_{r}$ must be zero. The derivative of either of these equations with respect to $x_{r}$ gives

$$
0=1-\left(\frac{p_{x}}{p}\right)^{2}-\frac{t}{p^{2}} p_{x x}
$$

where $p_{x x}$ denotes the derivative of $p_{x}$ with respect to $x_{r}$; namely,

$$
p_{x x}=\frac{\partial p_{x}}{\partial x_{r}}=\frac{\partial^{2} t}{\partial x_{r}^{2}}
$$

Solving equation 10 for $p$ gives

$$
p^{2}=p_{x}^{2}+t p_{x x}
$$

where $p$ is the effective slowness. To obtain the expressions for constant effective-velocity time migration, expression 12 is substituted in expressions 8 and 9.

This construction is based on a reflected signal and is not well defined for direct arrivals. For direct arrivals, the denominators in expressions 8 and 9 will vanish because for direct arrivals $t=p_{x}\left(x_{r}-x_{s}\right)+\sqrt{p^{2}-p_{x}^{2}}\left(z_{s}-z_{r}\right)$. This vanishing of the denominators effectively disregards the direct arrivals from 
the migration; there is no need for their removal before the migration.

In the appendix, I present the migration expressions generalized to three dimensions. To obtain the migration velocity, one does not have to use expression A-4; one could use analogous expressions obtained by considering the stationarity of the reflected image coordinates with respect to the change of $y_{r}$; namely,

$$
p^{2}=p_{y}^{2}+t p_{y y}
$$

The migration velocity is determined by the second derivative of the traveltime with respect to only one of the coordinates of the receiver. This means that it is not necessary to have the same data sampling in $x$ and $y$ directions to find the second derivative of the traveltime with respect to the location of receivers. To find the migration velocity, one can use only the derivatives along the direction with finer receiver spacing.

\section{EXAMPLES}

In the first example, I use the method to migrate synthetic data generated by the sufdmod 2 code from Seismic Unix (Cohen and Stockwell, 2007) from the velocity model used by Cooke et al. (2009) and shown in Figure 3. For brevity, I do not include a figure of the corresponding density model; the densities of the layers are in the descending order 1.0, 1.7, 1.8, 1.9, $3.0,2.4,3.0,2.25,3.2,2.25,3.0 \mathrm{~g} / \mathrm{cm}^{3}$. The wavelength is in the range of $40-100 \mathrm{~m}$.

To use the presented migration method, the following attributes of the shot gather are needed:

1) horizontal slowness

2) derivative of horizontal slowness

An example of a shot gather is shown in Figure 4. In this work, I use the direction of the gradient as an approximation of the local slope. In particular, the expression to compute the horizontal slowness is given by

$$
p_{x}=-\frac{\frac{\partial A}{\partial t}}{\frac{\partial A}{\partial x_{r}}},
$$

where $A(t, x)$ corresponds to the amplitude of the shot gather and the minus sign ensures that $p_{r}$ is positive for increasing traveltime with offset. The second derivative of the traveltime with offset can be calculated as a directional derivative of $p$ along the constant amplitude curve scaled by the projection of this direction to the offset axis. In particular, the resulting expression is

$$
p_{x x}=\frac{\partial p_{x}}{\partial x_{r}}+p_{x} \frac{\partial p_{x}}{\partial t}
$$

In a discretized case, one can use the central difference approximation of the partial derivatives. An example of the resulting horizontal slowness is given in Figure 5 for a fixed source at location $x_{s}=3900 \mathrm{~m}$. I choose to smooth the horizontal slowness by convolving with a $100 \mathrm{~m} \times 5 \mathrm{~ms}$ boxcart function to improve the quality of the second derivatives, which are illustrated in Figure 6.

Using equations 12 and 8, I compute the attributes corresponding to the migration velocity and the migration offset, respectively, for each event in a source gather. Figures 7 and 8 show these attributes for the source located at $3900 \mathrm{~m}$.

Equation 9 gives the zero-offset two-way traveltime attribute, which is displayed in Figure 9 for the same source gather as the other presented attributes. The migrated image of a single shot gather is given in Figure 10. Figure 11 shows the combination of all 60 source gathers. Figure 12 shows the migration velocity.

In the second example, I show the behavior of the method when applied to nonplanar reflectors with scatter points. Figure 13 shows the velocity model that was used to generate the synthetic shot gathers by Seismic Unix. Figure 14 shows the migrated events corresponding to different source-receiver pairs.

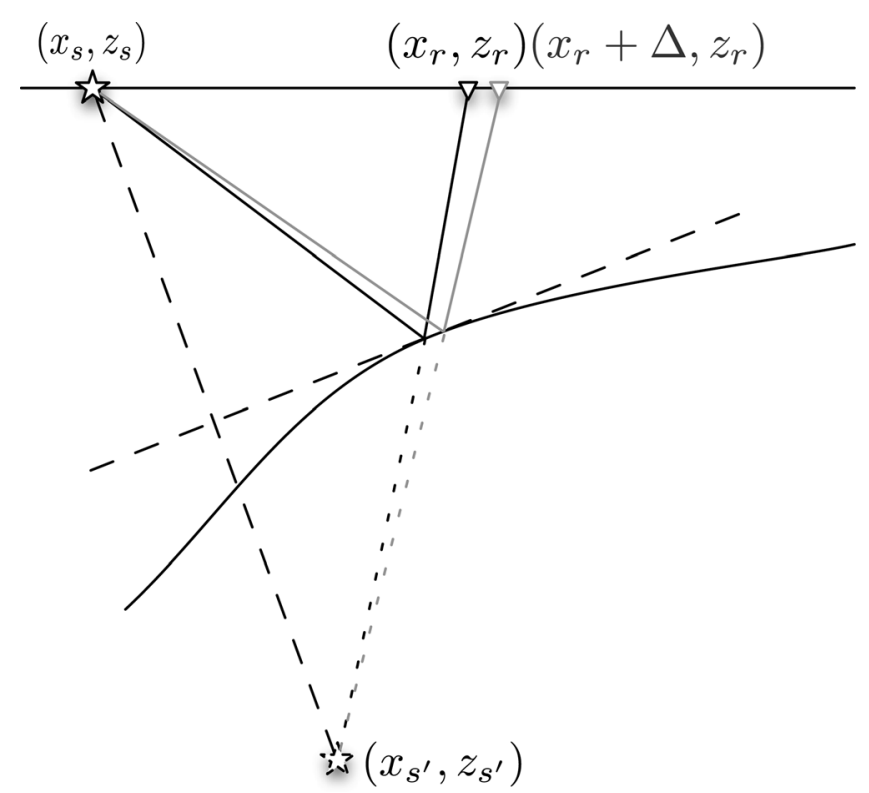

Figure 2. Stationarity of the reflected image with changing receiver location.

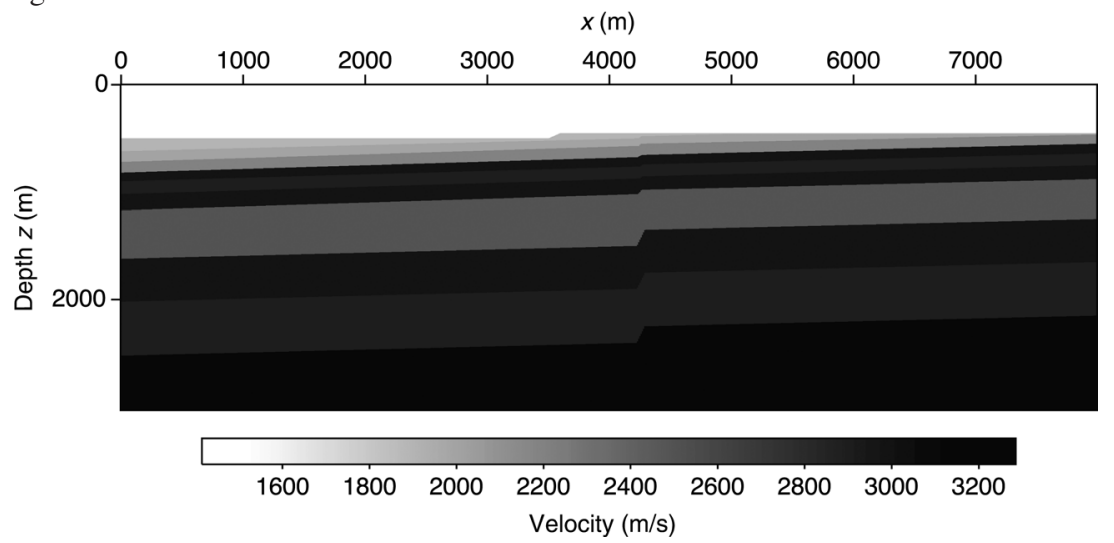

Figure 3. P-wave interval velocity model used for data generation. 


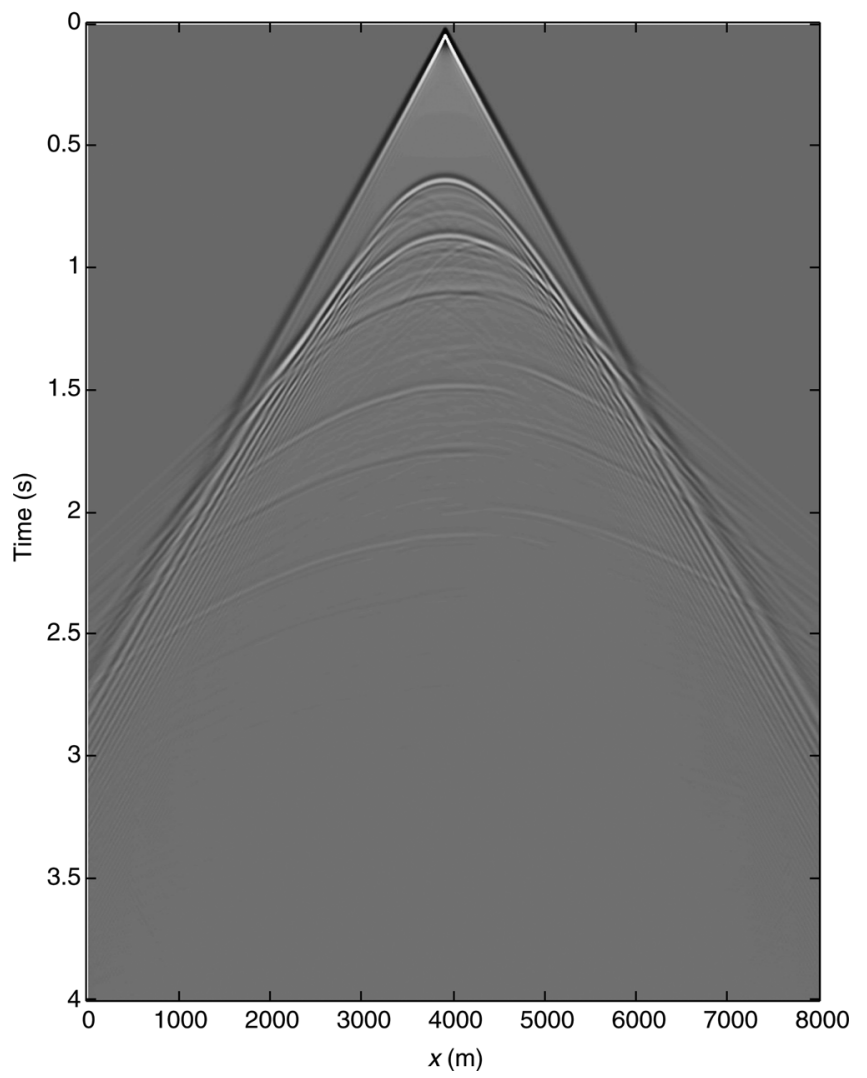

Figure 4. Example of a source gather at $3900 \mathrm{~m}$ obtained with the velocity model of Figure 3.

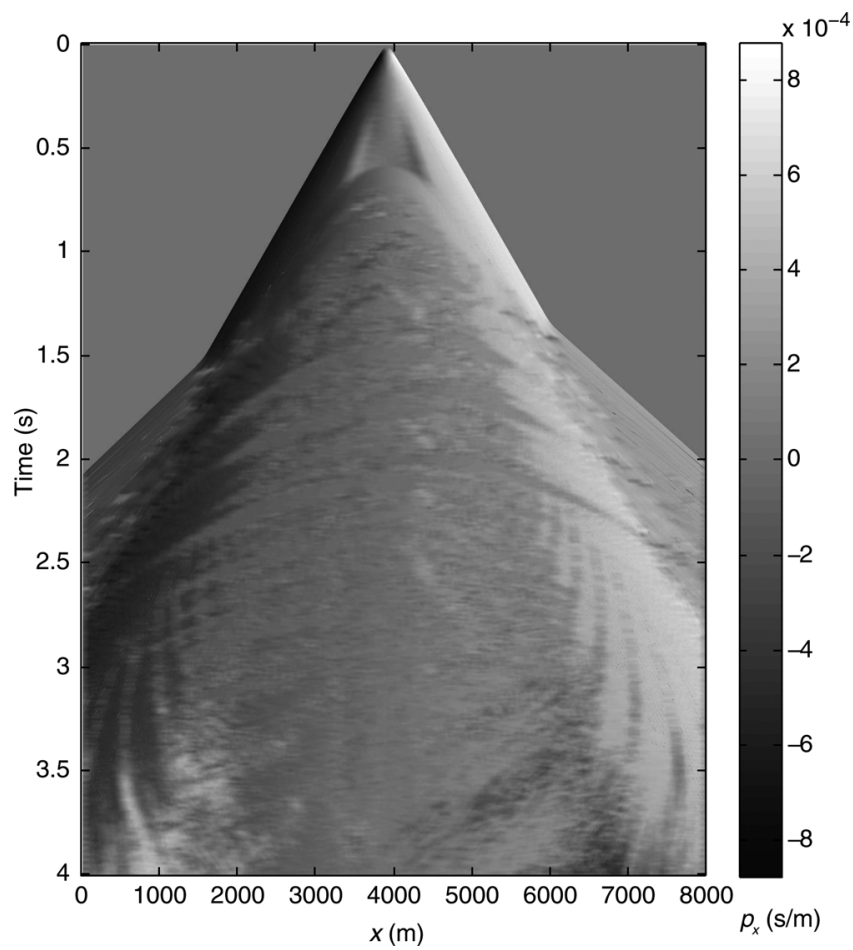

Figure 5. Horizontal slowness obtained from the shot gather in Figure 4.

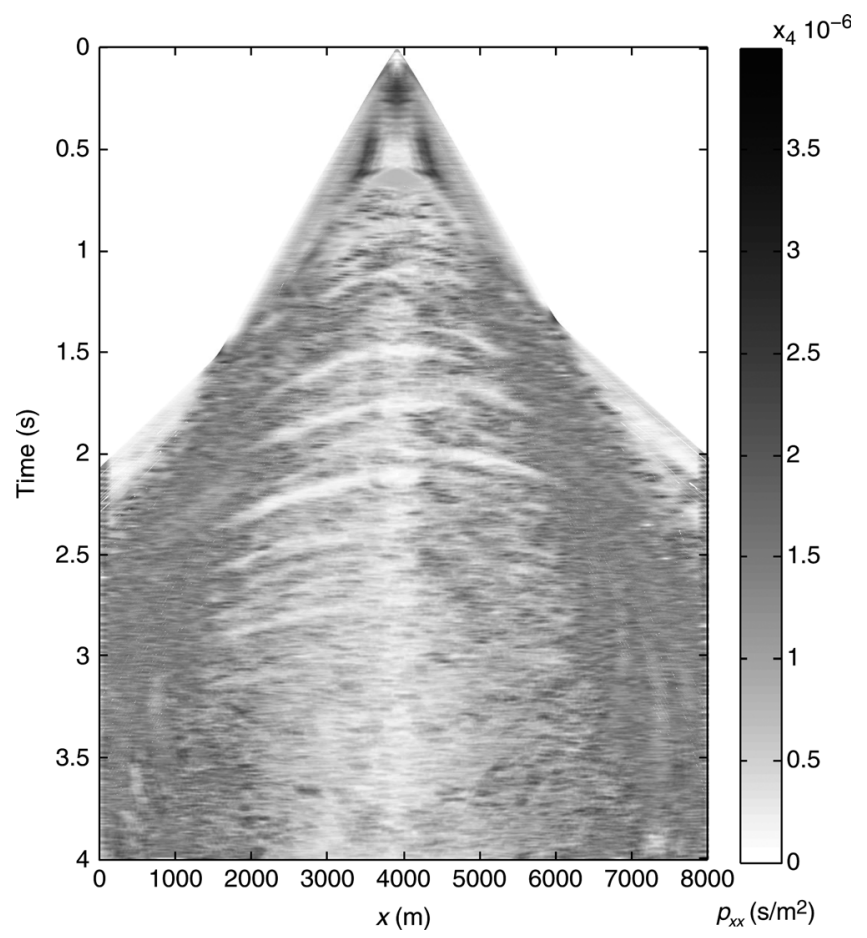

Figure 6. Second derivative of traveltime obtained from the shot gather in Figure 4.

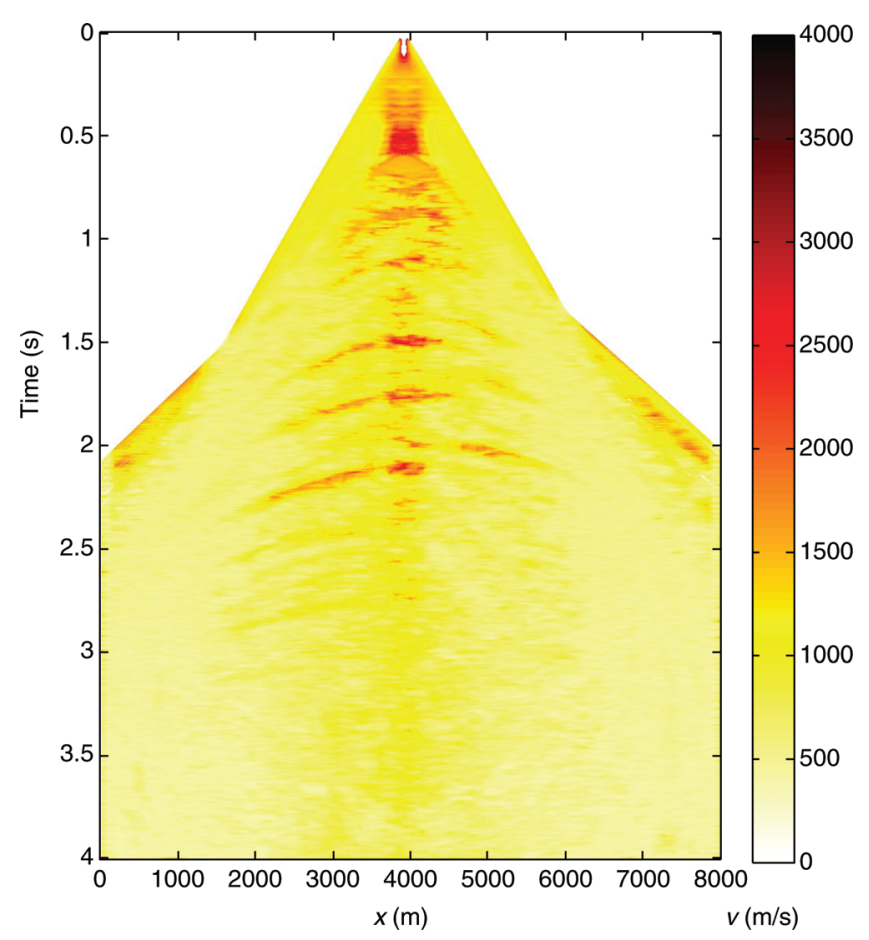

Figure 7. Migration velocity attribute obtained from the shot gather in Figure 4. 
It can be seen that the planar reflectors are well imaged, whereas the curvature of the nonplanar reflectors causes blurring of the image (indicated by arrows in Figure 14). This fact can be better

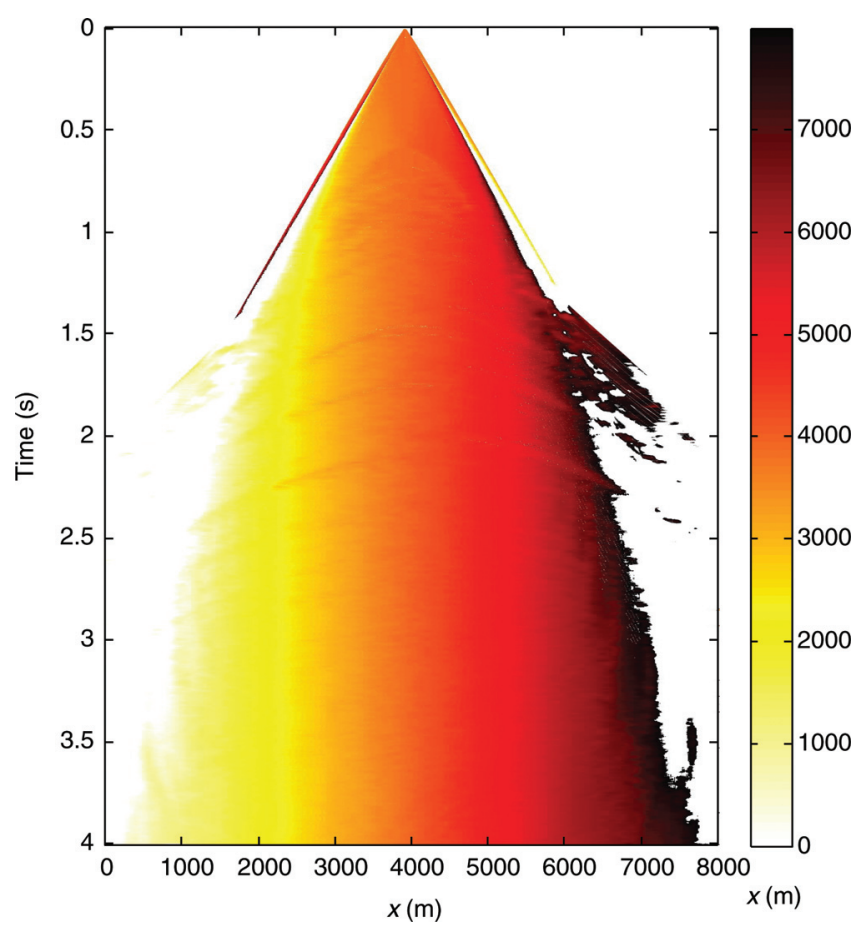

Figure 8. Migration offset attribute obtained from the shot gather in Figure 4. demonstrated by comparing two migrated shot gathers: one above the flat part of the reflectors (Figure 15) and one above the curved part of the reflectors (Figure 16). The least focused part of

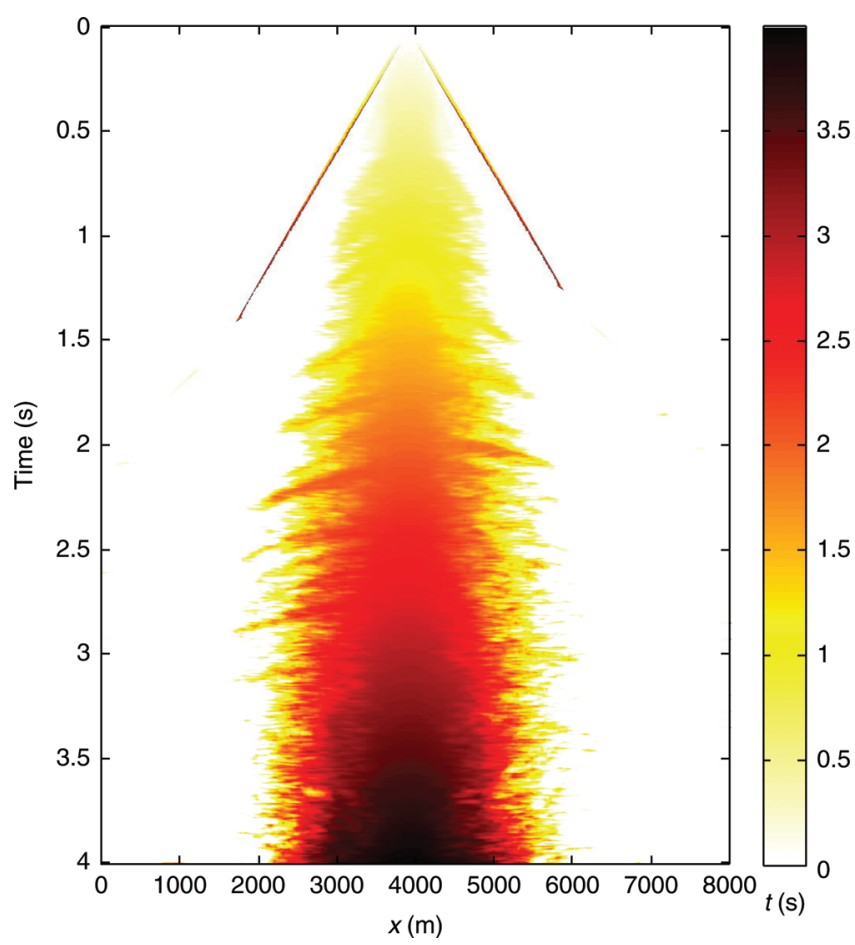

Figure 9. Migration two-way traveltime obtained from the shot gather in Figure 4.
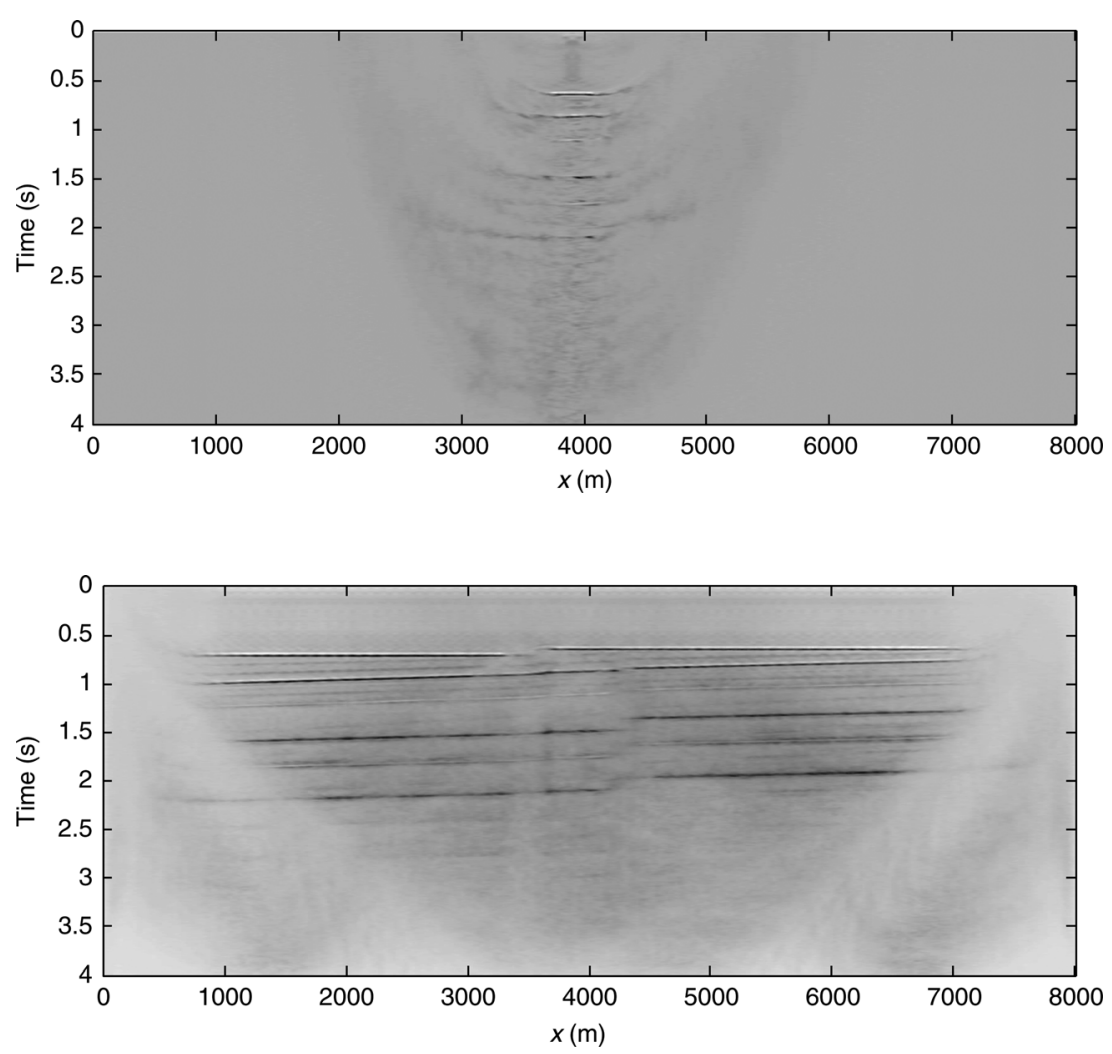

Figure 10. Migrated source gather obtained from the shot gather in Figure 4.

Figure 11. Image composed of 60 migrated source gathers with shots at every $100 \mathrm{~m}$ starting at $x=1000 \mathrm{~m}$ for the velocity model given in Figure 3. 


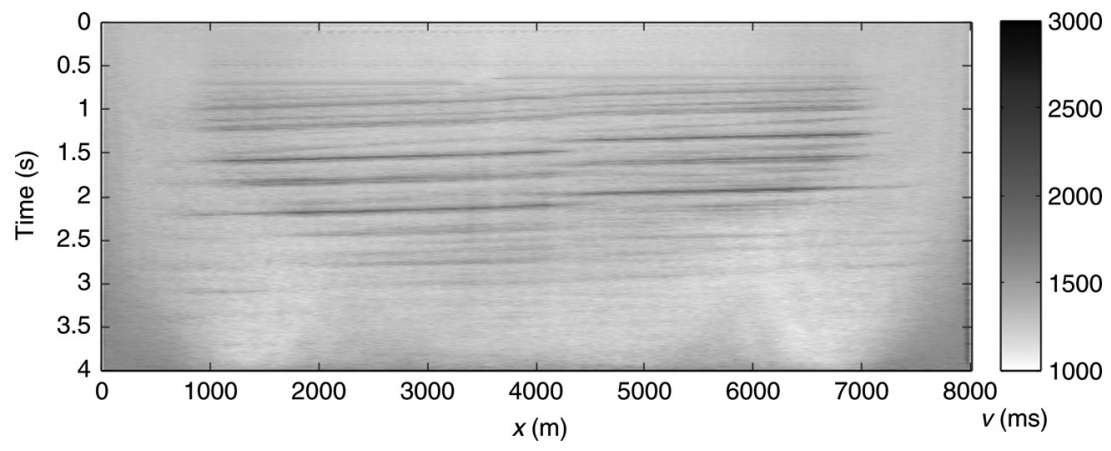

Figure 12. Migration velocity of 60 migrated source gathers.

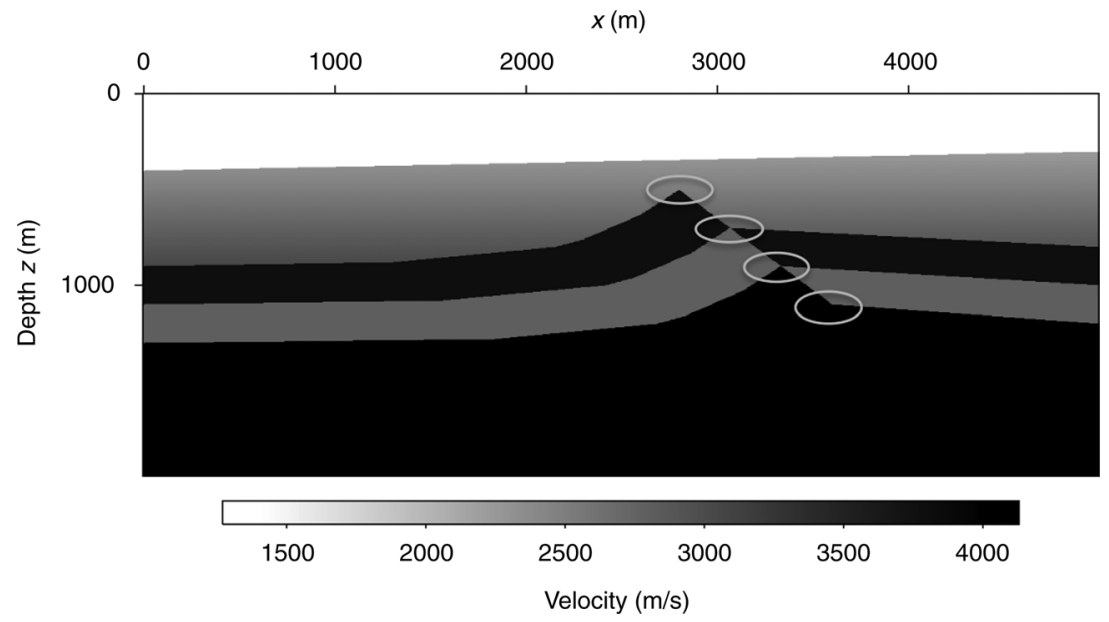

Figure 13. P-wave interval velocity model for testing migration of nonplanar reflectors. The ellipses indicate the location of scatter points.

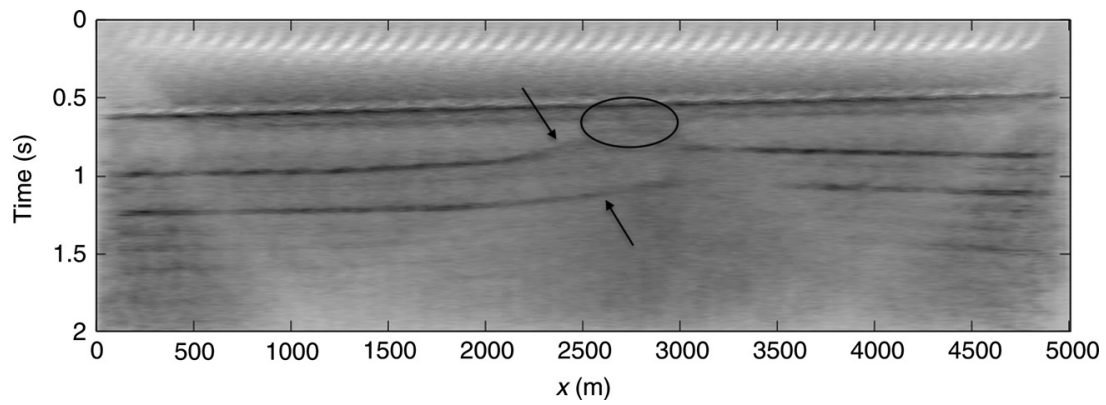

Figure 14. Combination of migrated source gathers in the velocity model of Figure 13 using the assumption of linear reflectors. The ellipse indicates the location of a scatter point and the arrows indicate the locations of the blurred curved reflectors.

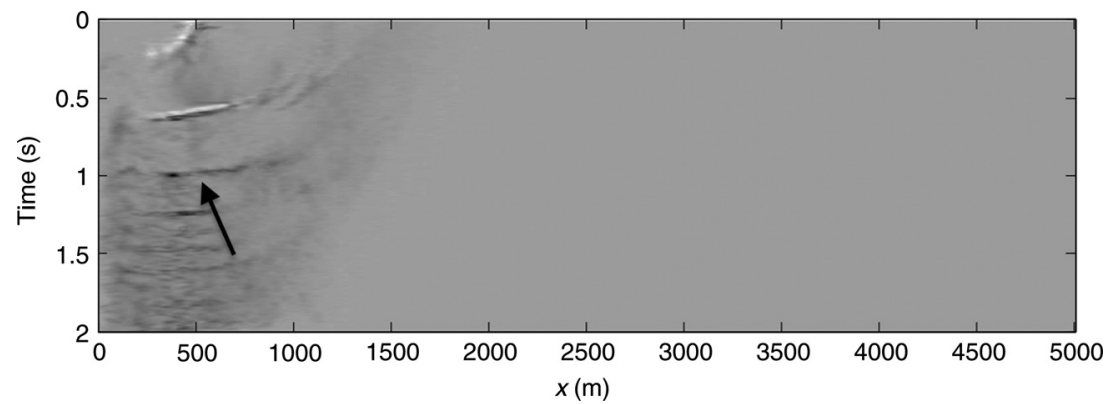

Figure 15. Migrated source gather with the source located at $400 \mathrm{~m}$ in the velocity model of Figure 13. The arrow indicates the location of a planar reflector. the image corresponds to the scatter points, indicated by the ellipse for the uppermost reflector. Note that the amplitude of the diffracted waves is of the same order as the amplitude of the waves reflected from the corresponding reflectors.

In the third example, I use a point scatterer in a homogeneous medium to generate synthetic source gathers. The resulting migration, together with the point scatterer, is shown in Figure 19. This example illustrates the defocusing of events that are the results of scattering. This lack of good focusing is due to the infinite curvature of the point scatterers and represents the worst conditions for the presented method that assumes zero curvature of the reflectors. The nonzero curvature affects the velocity estimates and the migration locations.

\section{DISCUSSION}

To assess the applicability of the proposed method for nonconstant true velocity above the reflector, I follow the development of Cameron et al. (2007), who looked at the relation between the time-migration velocities and the true seismic velocities by expanding the traveltime in the Taylor series. Unlike their approach of expanding the traveltime around the normal ray, I expand the traveltime around the source location. This difference is due to the use of the shot gathers in the present method. The first terms of the Taylor series of the square of the traveltime are

$$
\begin{aligned}
t^{2}(h)= & t^{2}(0)+2 \frac{\partial t(0)}{\partial h} t(0) h \\
& +\left(\left(\frac{\partial t(0)}{\partial h}\right)^{2}+t(0) \frac{\partial^{2} t(0)}{\partial h^{2}}\right) h^{2} \\
& +O\left(h^{3}\right) .
\end{aligned}
$$

The presented migration assumes that the traveltime is of the form

$$
t^{2}(h)=t^{2}(0)+2 t(0) h p \cos \gamma+p^{2} h^{2},
$$

where the traveltime is exact for a physical situation with a constant velocity above the planar interface, and $\gamma$ is the emergence angle of the zero-offset ray, measured from the surface. Using the notation from the previous sections, one can see that these formulas are identical to the second order of $h$ for the migration slowness satisfying expression 12 evaluated at the zero offset; namely, 


$$
p^{2}=p_{x}^{2}(0)+t(0) p_{x x}(0) .
$$

This result shows that the migration velocity $\frac{1}{p}$ is given by the shape of the traveltime curve in the vicinity of the zero offset for each source gather. If this velocity does not change much from shot gather to shot gather, then the presented imaging is a time migration with the migration velocity corresponding to this constant effective velocity. For laterally homogeneous media, this velocity becomes the Dix root mean square (rms) velocity. The Taylor expansion also highlights the complexity of the application of the method to 3D; the method assumes constant stacking velocity $\frac{1}{p}$ that might not satisfy the corresponding expressions using $p_{y}$ and $p_{y y}$ for nonconstant true velocity above the reflector. However, for some situations (e.g., marine seismics) the use of the migration velocity obtained along one direction might give satisfactory results.

The presented method of the first and second derivative estimation required smoothing of the data. However, there are many different methods for estimation of these parameters that are sensitive to the noise to a different degree. Some of the methods for estimation of local slopes include traditional slant stacks proposed by Ottolini (1983), plane-wave destructors used by Claerbout (2004), prediction-error filters suggested by Fomel (2002), Barnes (1996) uses instantaneous frequency, and Douma and de Hoop (2007) propose curvelets. Some of these methods are compared by Schleicher et al. (2009). Methods for the estimation of the second derivatives are less numerous; semblance analysis following the work of Taner and Koehler (1969) is the most common. The study of different methods for estimation of the second derivatives in the presence of noise is still an open problem; however, to illustrate the effects of noise on the method I polluted the data from the model from Figure 13 with random noise (Figure 17) with signal-to-noise ratio of 40 (computed at the maximum amplitude of the gathers). The result of the migration algorithm is shown in Figure 18.

A central assumption of the presented method is that the reflectors are planar; the radius of the curvature of the reflector should be much larger than the distance from the source to the reflector. The effect of the reflector curvature on the migration is such that for convex reflectors the migrated image is above the true reflector, and for concave reflectors the migrated image is below the true reflector. This behavior is exemplified by the infinitely curved convex reflector - a

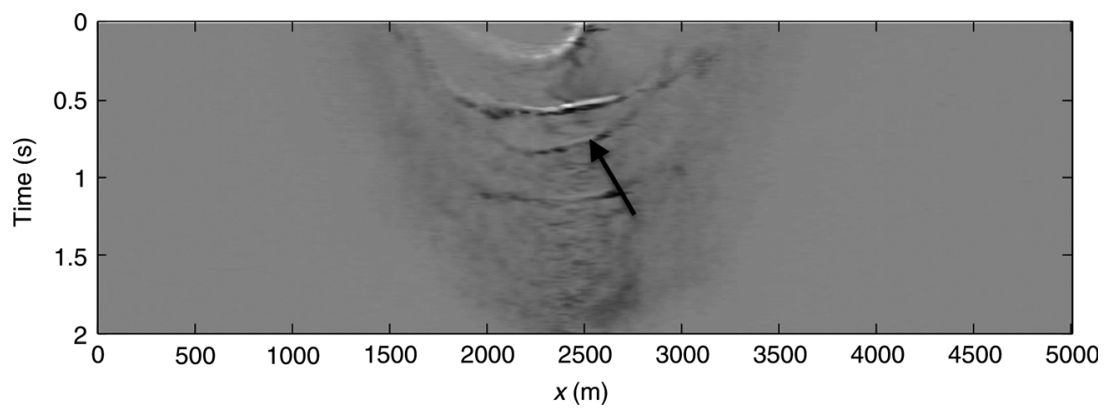

Figure 16. Migrated source gather with the source located at $2400 \mathrm{~m}$ in the velocity model of Figure 13. The arrow indicates the location of a curved reflector.

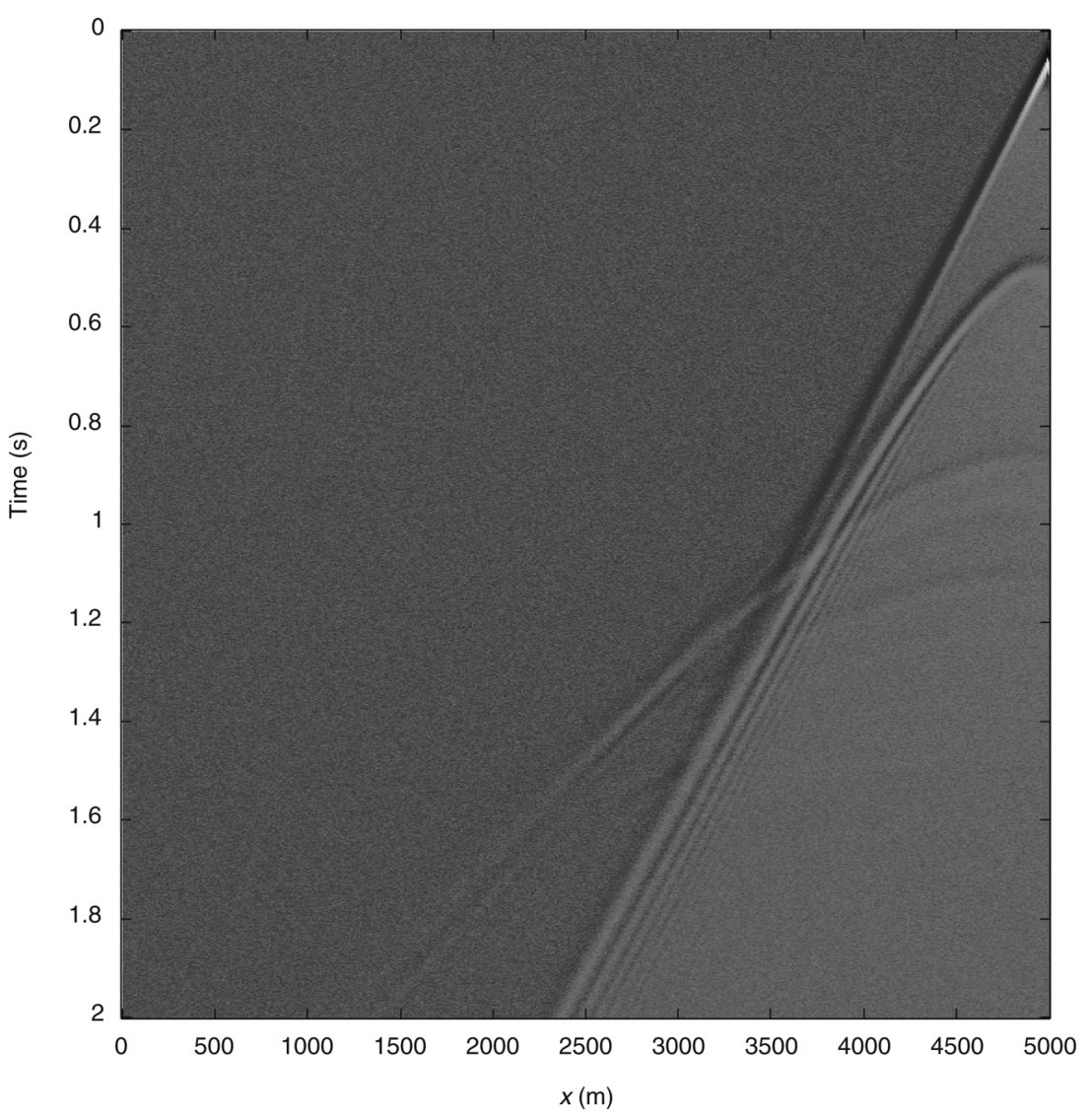

Figure 17. Source gather for the velocity model of Figure 13 with added random noise.

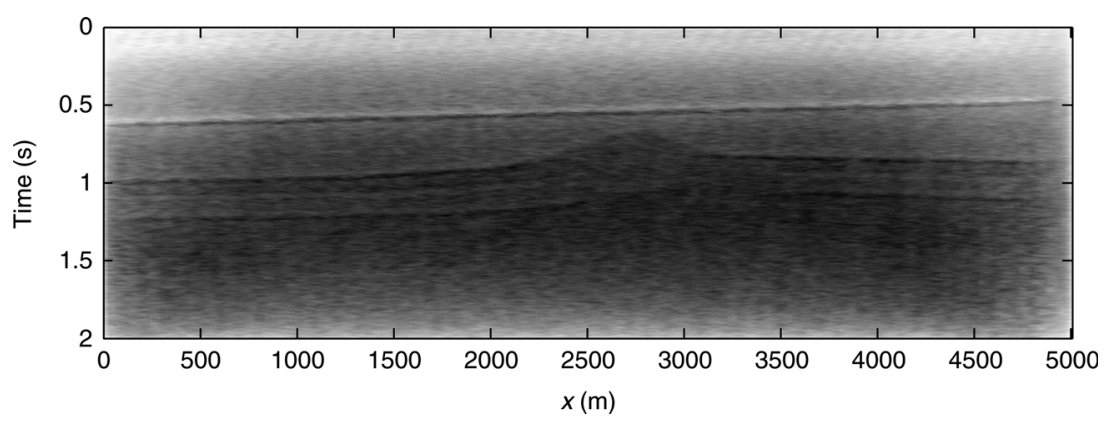

Figure 18. Migrated source gathers in the velocity model of Figure 13 with added random noise. 

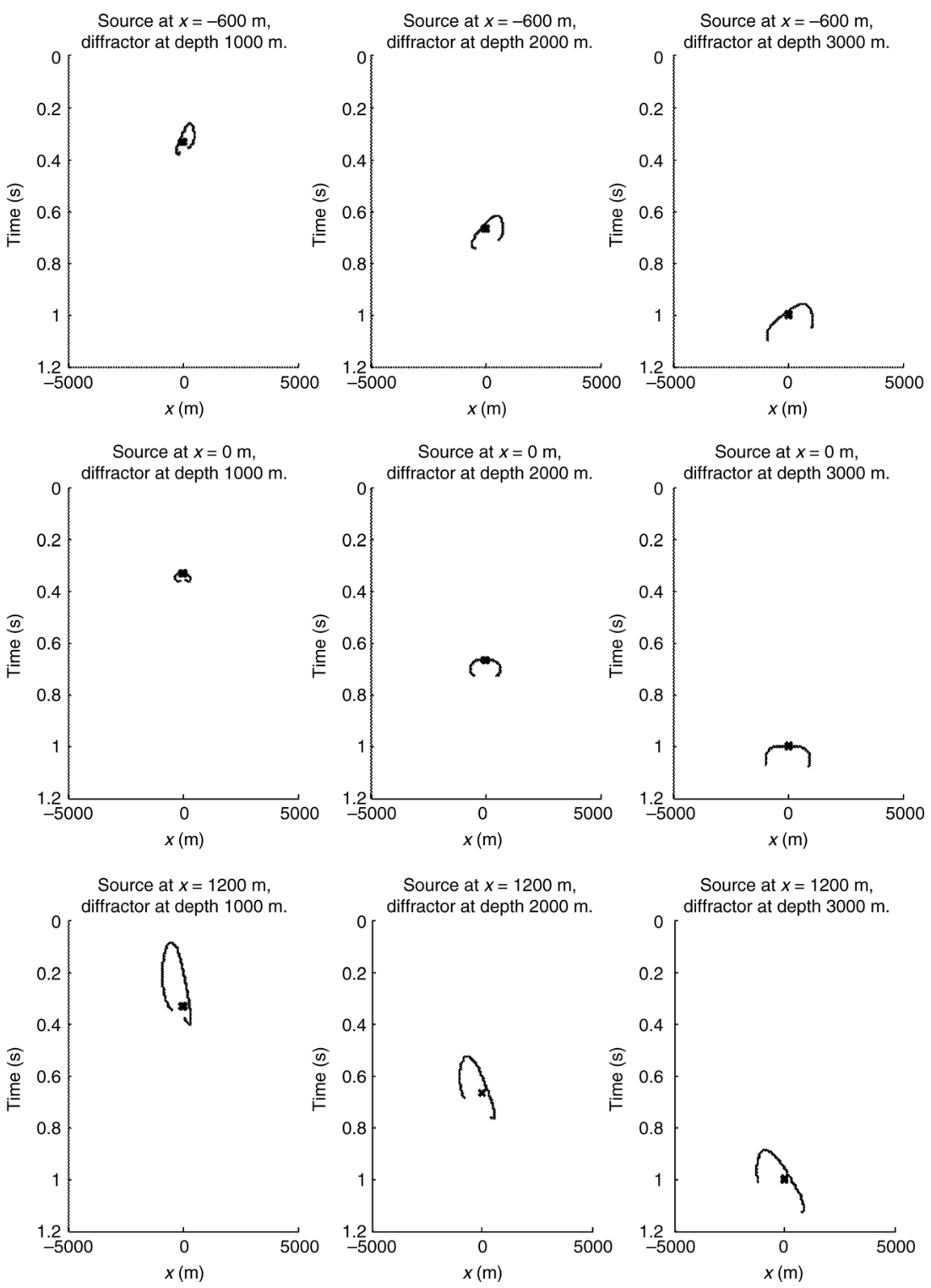

Figure 19. Migrated image of a shot gather of a point scatterer (curve) and the correct location of the point scatterer (cross).

scatter point; the migrated image of a shot gather corresponding to a scatter point is shown in Figure 19. Note that the shown migrated image contains no information about amplitudes because it is based only on the traveltime; it does not show the decrease in the amplitude of the events with increasing offset of the migrated event. This inability to correctly focus scatter points could be used potentially for separation of reflectors from scatter points. However, such a separation would have to be evaluated and compared with prior work on the subject (e.g., Harlan et al., 1984; Landa and Keydar, 1998; Fomel et al., 2007).

Because the method migrates the data from a shot gather and its implementation is very fast, one possible application for the method is imaging during the acquisition. Another possible application of the method — after simple modification — is for velocity seismic profile (VSP) situations. The limitation of the small curvature of the reflector prevents the applicability of the method to geological settings such as salt domes, where one would use depth migration anyway.

\section{CONCLUSIONS}

I presented a velocity-independent prestack migration of a source gather that uses the first and second derivatives of traveltime with respect to the location of the receivers. The assumptions of the constant effective velocity and locally planar reflector will affect the applicability of the algorithm only to certain geological situations, as is demonstrated by the second presented example. The presented method relies only on shot gathers and has several advantages over other velocity-independent migration methods: The method is computationally fast and requires little memory. In addition, the speed and the limited data requirements of the algorithm allow for potential real-time imaging during acquisition in the field. 
The inability of the method to focus diffracted events, as demonstrated by the third presented example, could be potentially used to separate scatter points from reflectors. Such a differentiation between scatter points and reflectors will be part of future studies, which will also include multiples suppression and extending the method for interfaces with nonzero curvature. In addition, it is important to study different methods for estimation of the second derivatives of the traveltime in the presence of noise.

\section{ACKNOWLEDGMENTS}

I thank Sergey Fomel, two anonymous reviewers, and the associate editor, Isabelle Lecomte, for their comments and suggestions that considerably improved the paper. I also acknowledge the developers and the maintainer of SU, John Stockwell, for making available this valuable tool. SU stands for CWP/SU: Seismic Un ${ }^{*} \mathrm{x}$, a processing line developed at the Colorado School of Mines that is partially based on Stanford Exploration Project (SEP) software.

\section{APPENDIX A}

\section{D migration expressions}

In this appendix I present the generalization of migration equations 8,9 , and 12 for the $3 \mathrm{D}$ case. The derivation of these equations follows the derivation of equations 8 and 9 , and I only show the final result; namely,

$$
\begin{aligned}
x= & \frac{2 p^{2} x_{r}\left(t+p_{y}\left(y_{s}-y_{r}\right)+\sqrt{p^{2}-p_{x}^{2}-p_{y}^{2}}\left(z_{r}-z_{s}\right)\right)}{2 p^{2} D} \\
& +\frac{p_{x}\left(p^{2}\left(x_{s}^{2}-x_{r}^{2}+\left(y_{s}-y_{r}\right)^{2}+\left(z_{r}-z_{s}\right)^{2}\right)-t^{2}\right)}{2 p^{2} D} \\
y= & \frac{2 p^{2} y_{r}\left(t+p_{x}\left(x_{s}-x_{r}\right)+\sqrt{p^{2}-p_{x}^{2}-p_{y}^{2}}\left(z_{r}-z_{s}\right)\right)}{2 p^{2} D} \\
& +\frac{p_{y}\left(p^{2}\left(y_{s}^{2}-y_{r}^{2}+\left(x_{s}-x_{r}\right)^{2}+\left(z_{r}-z_{s}\right)^{2}\right)-t^{2}\right)}{2 p^{2} D} \\
t_{0}= & \frac{t^{2}-p^{2}\left(\left(x_{s}-x_{r}\right)^{2}+\left(y_{s}-y_{r}\right)^{2}+z_{s}^{2}-z_{r}^{2}\right)}{p D\left(p^{2}-p_{x}^{2}-p_{y}^{2}\right)^{-1 / 2}} \\
& +\frac{2 p^{2} z_{r}\left(t-p_{x}\left(x_{r}-x_{s}\right)-p_{y}\left(y_{r}-y_{s}\right)\right)}{p D},
\end{aligned}
$$

and

$$
p^{2}=p_{x}^{2}+t p_{x x}
$$

where $D$ is short for

$$
t+p_{x}\left(x_{s}-x_{r}\right)+p_{y}\left(y_{s}-y_{r}\right)+\sqrt{p^{2}-p_{x}^{2}-p_{y}^{2}}\left(z_{r}-z_{s}\right) .
$$

These expressions reduce to the corresponding expressions in the $2 \mathrm{D}$ case if one sets the $y$ coordinates of the source and receiver, together with $p_{y}$, to zero.

\section{REFERENCES}

Barnes, A. E., 1996, Theory of 2-D complex seismic trace analysis: Geophysics, 61, 264-272.

Cameron, M. K., S. Fomel, and J. A. Sethian, 2007, Seismic velocity estimation from time migration: Inverse Problems, 23, 1329-1369.

Claerbout, J. F., 2004, Earth sounding analysis: Processing versus inversion: Blackwell Scientific Publications.

Cohen, J. K., and J. J. W. Stockwell, 2007, CWP/SU: Seismic Un ${ }^{*}$ x release no. 40: An open source software package for seismic research and processing. Center for Wave Phenomena, Colorado School of Mines.

Cooke, D., A. Bóna, and B. Hansen, 2009, Simultaneous time imaging, velocity estimation and multiple suppression using local event slopes: Geophysics, 74, no. 6, WCA65-WCA73.

Douma, H., and M. de Hoop, 2007, Leading-order seismic imaging using curvelets: Geophysics, 72, no. 6, S231-S248.

Fomel, S., 2002, Applications of plane-wave destruction filters: Geophysics, 67, 1946-1960.

_ 2007, Velocity-independent time-domain seismic imaging using local event slopes: Geophysics, 72, no. 3, S139-S147.

Fomel, S., E. Landa, and M. T. Taner, 2007, Poststack velocity analysis by separation and imaging of seismic diffractions: Geophysics, 72, no. 6 , U89-U94.

Goldin, S. V., 1986, Seismic traveltime inversion: SEG.

Harlan, W. S., J. F. Claerbout, and F. Rocca, 1984, Signal/noise separation and velocity estimation: Geophysics, 49, 1869-1880.

Hermont, A., 1979, Letter to the editor, Re: Seismic controllable directional reception as practiced in the U.S.S.R.: Geophysics, 44, 1601-1602.

Kleyn, A. H., 1977, On the migration of reflection time contour maps: Geophysical Prospecting, 25, 125-140.

Landa, E., and S. Keydar, 1998, Seismic monitoring of diffraction images for detection of local heterogeneities: Geophysics, 63, 1093-1100.

Ottolini, R., 1983, Velocity independent seismic imaging: Stanford Exploration Project, 37, 59-68.

Schleicher, J., J. C. Costa, L. T. Santos, A. Novais, and M. Tygel, 2009, On the estimation of local slopes: Geophysics, 74, no. 4, P25-P33.

Sword, C. H., 1981, Controlled directional receptivity - A Russian method of pre-stack migration: Technical Report SEP-26: 289-296, Stanford Exploration Project.

$\ldots$, 1987, Tomographic determination of interval velocities from reflection seismic data: The method of controlled directional reception: Ph.D. thesis, Stanford Exploration Project, Stanford University.

Taner, M. T., and F. Koehler, 1969, Velocity spectra - Digital computer derivation applications of velocity functions: Geophysics, 34, 859-881.

Zavalishin, B. R., 1982, Improvements in constructing seismic images using the method of controlled directional reception: 52nd Annual International Meeting, SEG, Expanded Abstracts, 1, 121-122, doi:10.1190/ 1.1826844 . 